\title{
CLINICAL IMAGE QUALITY EVALUATION OF MAMMOGRAPHY FOR BREAST CANCER SCREENING
}

Flávia Vidal Cabero', Ruffo Freitas-Junior², Rosângela da Silveira Corrêa³, Danielle Cristina Netto Rodrigues', Leonardo Ribeiro Soares², Paola Ferreira de Freitas ${ }^{4}$

'Brazilian Breast Cancer Research Network, Mastology Program, Hospital das Clínicas, Universidade Federal de Goiás Goiânia (GO), Brazil.

²Faculty of Medicine, Hospital das Clínicas, Universidade Federal de Goiás - Goiânia (GO), Brazil.

${ }^{3}$ National Nuclear Energy Commission/Center for Nuclear Sciences in the Midwest - Goiânia (GO), Brazil.

${ }^{4}$ Resident of the Mastology Program of the Women's Health Reference Center , Hospital Pérola Byington - São Paulo (SP), Brazil.

Objectives: To evaluate mammographic image quality in a real clinical practice scenario for breast cancer screening. Materials and Methods: Observational prospective study where images from digital mammograms from Diagnostic Service (DS) in the state of Goiás in 2019 were analyzed. A specific protocol was created based on evaluation criteria of the Brazilian College of Radiology, European Guidelines, and American College of Radiology. For each variable, score 1 was attributed to conformity and zero for nonconformity. Logistic regression model was utilized using the following independent variables: location (city vs. country), Public health system - Sistema Unico de Saude (SUS) (public vs. private), number of monthly examinations ( $\leq 300$ vs. $>300$ ), device manufacturing year ( $\leq 2011$ vs. $>2011$ ), and breast density $(\leq 75 \%$ vs. $>75 \%$ of the parenchyma). Results: Of the 163 fully functioning mammograms, 151 (92.6\%) were eligible, providing 1,024 images. We evaluated 12,032 items, of which 4,096 were craniocaudal projections, 4,608 mediolateral oblique projections, and 3,328 equipment related. On the clinical image analysis as to the positioning of the patient, the higher conformity for symmetry parameters, in both projections ( $>90 \%)$, was observed. The conformity rate among the other parameters varied from $18.6 \%$ to $100 \%$. In the multivariable analysis, it was observed that only the variables monthly examinations (OR 3.44; 95\%CI 1.67-7.09; $\mathrm{p}=0.0008$ ) and mammogram device manufacturing year (OR 2.46; 95\%CI 1.02-5.95; $\mathrm{p}=0.04$ ) were related to a higher conformity rate. After the percentage consolidation conformity rate per DS, as to the final clinical mammography quality, no DS presented desirable conformity (>90\%), 20 DS obtained acceptable conformity (between $70 \%$ and $89 \%$ ), and $10 \mathrm{DS}$ presented conformity below 70\%. Conclusion: Conformity rate of mammographic examination is extremely low and varies according to the multiple parameters analyzed. Mammographies performed at centers with less productivity ( $\leq 300 /$ monthly) and with newer devices $(>2,011)$ presented higher chances of conformity at the clinical imaging evaluation.

Keywords: Screening; Mammography; Clinical Quality. 